\title{
Raman light scattering in pseudospin-electron model at strong pseudospin-electron interaction
}

\author{
T.S.Mysakovych, I.V.Stasyuk \\ Institute for Condensed Matter Physics \\ of the National Academy of Sciences of Ukraine, \\ 1 Svientsitskii Str., 79011 Lviv, Ukraine
}

Received May 5, 2004

Anharmonic phonon contributions to Raman scattering in locally anharmonic crystal systems in the framework of the pseudospin-electron model with tunneling splitting of levels are investigated. The case of strong pseudospin-electron coupling is considered. Pseudospin and electron contributions to scattering are taken into account. Frequency dependences of Raman scattering intensity for different values of model parameters and for different polarization of scattering and incident light are investigated.

Key words: Raman scattering, pseudospin-electron model

PACS: $71.10 \mathrm{Fd}, 71.10 . \mathrm{Fd}, 72.10 . \mathrm{Di}, 74.25 . \mathrm{KC}$

\section{Introduction}

This article is dedicated to the memory of Prof. Z. Gurskii, famous scientist and good friend, it was always pleasant and useful to communicate with him.

Raman scattering of light is a powerfull instrument in investigating the elementary excitations in different systems. In solids it is connected with energy transfer from light to crystal with the participation of low energy excitation, phonons or low energy transitions in electron subsystem. The problem of phonon and nonphonon (electron, magnon, plasmon and other) contributions to Raman light scattering in the systems with a strong short-range Hubbard-type interaction between electrons has been a subject of interest lately. Many investigations (both theoretical and experimental) were devoted to the study of Raman spectra in high-temperature superconductors (HTSC) (see review [1]) in order to better understand the nature of superconducting pairing mechanism and to explain the reason of complicated Raman spectra in HTSC. Raman spectra are also investigated for normal (nonsuperconducting) state for the cases of metals and dielectrics [2-4]. Resonance, nonresonance and mixed contributions to Raman spectra are studied [3-5]. 
In this work we calculate Raman spectra for the system described by the pseudospin-electron model (PEM) using the method which is based on the construction of a polarizability operator $\hat{P}$ in the framework of a microscopic approach; the method was developed in [6-9] and is an alternative to standard ones [2-5]. PEM describes the systems with locally anharmonic elements (anharmonic vibrations of apical oxygen $\mathrm{O} 4$ in HTSC of $\mathrm{YBaCuO}$-type, the systems with hydrogen bonds, etc.). In [9] we considered contributions to Raman spectra for the $z z$ polarization case $(z$ is a direction of local vibrations and it is perpendicular to the layer in the case of layered crystals of $\mathrm{YBaCuO}$-type). Here we investigate the case of $x x+y y$ polarization and compare these two cases. Like in [9], we consider the strong coupling case, when the interaction constants in the PEM are large in comparison with the width of electron band.

We use the expression for cross-section of Raman light scattering written in the following form $([6-10])$ :

$$
\frac{\partial^{2} \sigma}{\partial \Omega \partial \omega_{2}}=\frac{1}{\left(4 \pi \varepsilon_{0}\right)^{2}} \sqrt{\frac{\varepsilon_{1}}{\varepsilon_{2}}} \frac{\omega_{2}^{3} \omega_{1}}{c^{4}} \sum_{\alpha \beta \alpha^{\prime} \beta^{\prime}} e_{1 \alpha} e_{2 \beta} e_{1 \alpha^{\prime}} e_{2 \beta^{\prime}} H_{\vec{k}_{2}, \vec{k}_{1}}^{\beta^{\prime} \alpha^{\prime}, \beta, \alpha}\left(\omega_{1}, \omega_{2}\right),
$$

hereafter we put $\hbar=1, \vec{e}_{1}, \vec{e}_{2}$ are polarization vectors; $\omega_{1}, \omega_{2}$ are incident and scattered light frequencies; $\vec{k}_{1}, \vec{k}_{2}$ are the corresponding wave vectors; $\varepsilon_{1,2} \equiv \varepsilon\left(\omega_{1}, \omega_{2}\right)$; $H_{\vec{k}_{2}, \vec{k}_{1}}^{\beta^{\prime} \alpha^{\prime}, \beta, \alpha}\left(\omega_{1}, \omega_{2}\right)$ is the Raman scattering tensor:

$$
H_{\vec{k}_{2},-\vec{k}_{1} ;-\vec{k}_{2}, \vec{k}_{1}}^{\beta^{\prime} \alpha^{\prime}, \beta,}\left(\omega_{1}, \omega_{2}\right)=\frac{1}{2 \pi} \int_{-\infty}^{+\infty} \mathrm{d} t \mathrm{e}^{\mathrm{i}\left(\omega_{1}-\omega_{2}\right) t}\left\langle\hat{P}_{\vec{k}_{2}-\vec{k}_{1}}^{\beta^{\prime} \alpha^{\prime}}\left(-\omega_{1}, t\right) \hat{P}_{-\vec{k}_{2} \vec{k}_{1}}^{\beta \alpha}\left(\omega_{1}, 0\right)\right\rangle,
$$

while $\hat{P}$ is the polarizability operator

$$
\hat{P}_{\vec{k}^{\prime} \vec{k}}^{\beta \alpha}(\omega, t)=-\int_{-\infty}^{+\infty} \mathrm{d} s \mathrm{e}^{\mathrm{i} \omega(t-s)}\left\{\left\{\hat{M}^{\beta}\left(\overrightarrow{k^{\prime}}, t\right) \mid \hat{M}^{\alpha}(\vec{k}, s)\right\}\right\} .
$$

Here $\hat{M}^{\alpha}(\vec{k})$ is a dipole moment of a crystal unit cell in the $\vec{k}$-representation and the symbol $\left\{\left\{\hat{M}^{\beta}\left(\overrightarrow{k^{\prime}}, t\right) \mid \hat{M}^{\alpha}(\vec{k}, s)\right\}\right\}$ stands for "unaveraged" Green function defined in the following way $([6,9])$ :

$$
\left\{\left\{A(t) \mid B\left(t^{\prime}\right)\right\}\right\}=-i \Theta\left(t-t^{\prime}\right)\left[A(t), B\left(t^{\prime}\right)\right] .
$$

The equations of motion for this function have a form

$$
\begin{aligned}
& \omega_{1}\{\{A \mid B\}\}_{\omega_{1}, \omega_{2}}=\frac{1}{2 \pi}[A, B]_{\omega_{1}-\omega_{2}}+\{\{[A, H] \mid B\}\}_{\omega_{1}, \omega_{2}}, \\
& \omega_{2}\{\{A \mid B\}\}_{\omega_{1}, \omega_{2}}=\frac{1}{2 \pi}[A, B]_{\omega_{1}-\omega_{2}}-\{\{A \mid[B, H]\}\}_{\omega_{1}, \omega_{2}} .
\end{aligned}
$$

They are used to construct the polarizability operator; the solutions of these equations are built in the form of operator series in powers of some parameters of a Hamiltonian. It should be emphasized that this method does not use phenomenological assumptions. 


\section{PEM with strong interaction}

PEM with tunneling splitting of levels is to some extent a generalization of the known Falicov-Kimball model [11] when the proper dynamics of the locally anharmonic elements of structure is present in the system. Pseudospin formalism is used to describe vibrations for the case of double-well potential. The Hamiltonian of the PEM is as follows:

$$
\begin{gathered}
H=\sum_{i}\left(U n_{i, \uparrow} n_{i, \downarrow}-\mu\left(n_{i, \uparrow}+n_{i, \downarrow}\right)+g S_{i}^{z}\left(n_{i, \uparrow}+n_{i, \downarrow}\right)\right. \\
\left.-h S_{i}^{z}-\Omega S_{i}^{x}\right)+\sum_{i, j, \sigma} t_{i j} a_{i, \sigma}^{\dagger} a_{j, \sigma} .
\end{gathered}
$$

It includes electron transfer from site to site $\left(t_{i j}\right.$-term), Coulomb interaction ( $U$ term), pseudospin-electron interaction ( $g$-term), tunnelling splitting of the vibrational mode $(\Omega$-term), asymmetry of local potential ( $h$-term) [12-14]. The investigations of the PEM revealed a possibility of phase transition to chess-board phase as well as incommensurate phase, the transition between uniform phases, the transition to a superconducting state, the existence of phase separation region and other peculiarities [15-20].

Let us introduce a single-site basis of states: $|i, Q\rangle \equiv\left|n_{i \uparrow}, n_{i \downarrow}, S_{i}^{z}=1 / 2\right\rangle, \mid i, \widetilde{Q}>\equiv$ $\mid n_{i \uparrow}, n_{i \downarrow}, S_{i}^{z}=-1 / 2>$,

$$
\begin{aligned}
& |i, 1 ; \widetilde{1}\rangle=\left|i, 0,0, \pm \frac{1}{2}\right\rangle,|i, 2 ; \widetilde{2}\rangle=\left|i, 1,1, \pm \frac{1}{2}\right\rangle, \\
& |i, 3 ; \widetilde{3}\rangle=\left|i, 0,1, \pm \frac{1}{2}\right\rangle,|i, 4 ; \widetilde{4}\rangle=\left|i, 1,0, \pm \frac{1}{2}\right\rangle .
\end{aligned}
$$

Using the transformation

$$
\begin{aligned}
& \left|i, Q>=\cos \phi_{r}\right| i, r>+\sin \phi_{r} \mid i, \widetilde{r}> \\
& \left|i, \widetilde{Q}>=\cos \phi_{r}\right| i, \widetilde{r}>-\sin \phi_{r} \mid i, r>, \\
& \cos 2 \phi_{r}=\frac{n_{r} g-h}{\sqrt{\left(n_{r} g-h\right)^{2}+\Omega^{2}}}, n_{1}=0 ; n_{2}=2 ; n_{3}=n_{4}=1,
\end{aligned}
$$

we diagonalize the single-site part of the Hamiltonian

$$
\begin{aligned}
H_{0} & =\sum_{i, r} \lambda_{r} X_{i}^{r r}+\sum_{i, \widetilde{r}} \lambda_{\widetilde{r}} X_{i}^{\widetilde{r}}, \\
\lambda_{r, \widetilde{r}} & =n_{r} \mu+U \delta_{r, 2} \pm \frac{1}{2} \sqrt{\left(n_{r} g-h\right)^{2}+\Omega^{2}} .
\end{aligned}
$$

Dipole moment of an unit cell is given by the expression

$$
M_{i}^{\alpha}=e R_{i}^{\alpha}\left(n_{\uparrow, i}+n_{\downarrow, i}\right)+d_{s} S_{i}^{z} \delta_{\alpha z},
$$

the local anharmonic double well is oriented along $z$ direction, $d_{s}$ is a dipole moment of pseudospins, $R_{i}^{\alpha}$ is a $\alpha$ component of the radius-vector $\vec{R}_{i}, e$ is an electron charge. 
We take into account the pseudospin and electron contributions to a dipole moment [9].

We construct the polarizability operator by means of the equation of motion method, using the expansion in terms of the electron transfer parameter. As the first step, we obtain (see for details [9]):

$$
\begin{aligned}
\left\{\left\{M_{k}^{\alpha} \mid M_{l}^{\beta}\right\}\right\}= & \frac{\delta_{k, l} \delta_{\alpha, z} \delta_{\beta, z} d_{s}^{2}}{8 \pi \omega_{1}} \sum_{r} \sin \left(4 \phi_{r}\right)\left(X_{k}^{r \widetilde{r}}-X_{k}^{\widetilde{r} r}\right) \\
& +\sum_{r} \frac{\delta_{k, l} \delta_{\alpha, z} \delta_{\beta, z} d_{s}^{2}}{8 \pi\left(\omega_{1}-\lambda_{r}+\lambda_{\widetilde{r}}\right)}\left(\sin \left(4 \phi_{r}\right) X_{k}^{\widetilde{r} r}-\sin ^{2}\left(2 \phi_{r}\right)\left(X_{k}^{r r}-X_{k}^{\widetilde{r r}}\right)\right) \\
& -\sum_{r} \frac{\delta_{k, l} \delta_{\alpha, z} \delta_{\beta, z} d_{s}^{2}}{8 \pi\left(\omega_{1}+\lambda_{r}-\lambda_{\widetilde{r}}\right)}\left(\sin \left(4 \phi_{r}\right) X_{k}^{r \widetilde{r}}-\sin ^{2}\left(2 \phi_{r}\right)\left(X_{k}^{r r}-X_{k}^{\widetilde{r r}}\right)\right) \\
& +\frac{e R_{k}^{\alpha}}{\omega_{1}} \sum_{i, j, \sigma} t_{i, j}\left(\delta_{i, k}-\delta_{j, k}\right)\left\{\left\{a_{i, \sigma}^{\dagger} a_{j, \sigma} \mid M_{l}^{\beta}\right\}\right\} \\
& +\sum_{r} \frac{d_{s} \delta_{\alpha, z}}{2 \omega_{1}} \sum_{i, j, \sigma} t_{i, j} \cos \left(2 \phi_{r}\right)\left\{\left\{\left[X_{k}^{r r}-X_{k}^{\widetilde{r} \widetilde{r}}, a_{i, \sigma}^{\dagger} a_{j, \sigma}\right] \mid M_{l}^{\beta}\right\}\right\} \\
& +\sum_{r} \frac{d_{s} \delta_{\alpha, z}}{2\left(\omega_{1}-\lambda_{r}+\lambda_{\tilde{r}}\right)} \sum_{i, j, \sigma} t_{i, j} \sin \left(2 \phi_{r}\right)\left\{\left\{\left[X_{k}^{\widetilde{r} r}, a_{i, \sigma}^{\dagger} a_{j, \sigma}\right] \mid M_{l}^{\beta}\right\}\right\} \\
& +\sum_{r} \frac{d_{s} \delta_{\alpha, z}}{2\left(\omega_{1}+\lambda_{r}-\lambda_{\widetilde{r}}\right)} \sum_{i, j, \sigma} t_{i, j} \sin \left(2 \phi_{r}\right)\left\{\left\{\left[X_{k}^{r \widetilde{r}}, a_{i, \sigma}^{\dagger} a_{j, \sigma}\right] \mid M_{l}^{\beta}\right\}\right\} .
\end{aligned}
$$

Let us consider the pure electron contributions to a Raman spectrum. For a corresponding term we can obtain the following expression

$$
\begin{aligned}
\sum_{i, j, \sigma} R_{k}^{\alpha} & R_{l}^{\beta} t_{i, j}\left(\delta_{i, k}-\delta_{j, k}\right)\left\{\left\{a_{i, \sigma}^{+} a_{j, \sigma} \mid\left(n_{\uparrow l}+n_{\downarrow l}\right)\right\}\right\}= \\
= & \frac{1}{\omega_{2}} R_{k}^{\alpha} R_{l}^{\beta} \sum_{\substack{i, j, \sigma \\
s, p, \sigma^{\prime}}} t_{i, j}\left(\delta_{i, k}-\delta_{j, k}\right) t_{s, p}\left(\delta_{p, l}-\delta_{s, l}\right)\left\{\left\{a_{i, \sigma}^{+} a_{j, \sigma} \mid a_{s, \sigma^{\prime}}^{+} a_{p, \sigma^{\prime}}\right\}\right\} \\
& -\frac{1}{2 \pi \omega_{2}} R_{k}^{\alpha} R_{l}^{\beta} \sum_{i, j, \sigma} t_{i, j}\left(\delta_{i, k}-\delta_{j, k}\right)\left(\delta_{i, l}-\delta_{j, l}\right) a_{i, \sigma}^{+} a_{j, \sigma} .
\end{aligned}
$$

In the long-wavelenght limit, $\vec{k}_{2}, \vec{k}_{1} \approx 0$, we perform summation over indexes $k, l$

$$
\begin{gathered}
-\sum_{k, l} \frac{1}{2 \pi \omega_{2}} R_{k}^{\alpha} R_{l}^{\beta} \sum_{i, j, \sigma} t_{i, j}\left(\delta_{i, k}-\delta_{j, k}\right)\left(\delta_{i, l}-\delta_{j, l}\right) a_{i, \sigma}^{+} a_{j, \sigma}= \\
=\frac{1}{2 \pi \omega_{2}} \sum_{q} \frac{\partial^{2} t(q)}{\partial q_{\alpha} \partial q_{\beta}} \alpha_{q}^{+} \alpha_{q}, \\
\alpha_{q}=\frac{1}{\sqrt{N}} \sum_{i} a_{i} \mathrm{e}^{-\mathrm{i} q R_{i}}, \quad t_{q}=\sum_{R} t(R) \mathrm{e}^{-\mathrm{i} q R} .
\end{gathered}
$$


The Raman scattering tensor can be expressed in terms of two-time Green functions $[9]$

$$
\begin{aligned}
& H_{k_{2}, k_{1}=0}^{\alpha \beta, \alpha^{\prime} \beta^{\prime}}\left(\omega_{1}, \omega_{2}\right)=\frac{-(2 \pi)^{2}}{\left(\mathrm{e}^{\beta \omega}-1\right)} \times \\
& \sum_{i, i_{1}, j, j_{1}} 2 \operatorname{Im}\left\langle\left\langle\left\{\left\{M_{i}^{\alpha} \mid M_{j}^{\beta}\right\}\right\}_{\omega_{2}, \omega_{1}} \mid\left\{\left\{M_{i_{1}}^{\alpha^{\prime}} \mid M_{j_{1}}^{\beta^{\prime}}\right\}\right\}_{-\omega_{2},-\omega_{1}}\right\rangle\right\rangle_{\omega+i \varepsilon}, \quad \omega=\omega_{2}-\omega_{1} .
\end{aligned}
$$

It should be noted that in contrast to other methods of Raman spectra investigations [5], in our approach many-time correlation functions do not appear; this fact essentially simplifies the calculation.

\section{Correlators in GRPA}

We investigate Raman spectra in the frequency region $\omega_{2}-\omega_{1}$ near the frequency $\omega^{\prime}=\lambda_{\widetilde{1}}-\lambda_{1}$. Correlators $\left\langle X^{p q}(t) X^{r s}\right\rangle$ are calculated using the method of temperature Green functions. The Hamiltonian is presented in the form

$$
\begin{aligned}
& H=H_{0}+H_{\mathrm{int}}, \\
& H_{\mathrm{int}}=\sum_{i, j, \sigma} t_{i, j} a_{i, \sigma}^{\dagger} a_{j, \sigma} .
\end{aligned}
$$

We consider two cases of polarization: i) light is polarized in $z z$ direction ii) light is polarized in $x x+y y$ direction. As it can be seen from the expression (12), in the

lowest order in $t_{i j}$ in the case of $z z$ polarization to obtain an expression for tensor $H\left(\omega_{1}, \omega_{2}\right)$ we should calculate Bose-type Green function, constructed on the $X^{r \tilde{r}}$, $X^{\widetilde{r} r}, X^{\widetilde{r} r}, X^{r r}$ operators

$$
\begin{aligned}
\left\langle T X_{l}^{p q}(\tau) X_{m}^{r s}\right\rangle & =K_{l m}^{p q r s}(\tau)=\left\langle T X_{l}^{p q}(\tau) X_{m}^{r s} \sigma(\beta)\right\rangle_{0}^{c}, \\
\sigma(\beta) & =T \exp \left(-\int_{0}^{\beta} H_{\mathrm{int}}(\tau) \mathrm{d} \tau\right) .
\end{aligned}
$$

Operators are given in the interaction representation $\mathrm{C}(\tau)=\mathrm{e}^{\tau H_{0}} \mathrm{Ce}^{-\tau H_{0}}$, the brackets $\langle\ldots\rangle_{0}$ stand for the statistical averaging with the Hamiltonian $H_{0}$, the symbol $\langle\ldots\rangle^{c}$ denotes the separation of connected diagrams. Expanding the exponent in powers of $H_{\text {int }}$, we obtain the averages of Hubbard operators; such averages are calculated using Wick's theorem [21]. After transformation to the momentum-frequency representation

$$
\int_{0}^{\beta} \mathrm{d} \tau \mathrm{e}^{\mathrm{i} \omega_{n} \tau} \frac{1}{N} \sum_{l m} K_{l m}^{p q r s}(\tau) \mathrm{e}^{\mathrm{i} q\left(R_{l}-R_{m}\right)}=K^{p q r s}\left(q, \omega_{n}\right) .
$$

The transition to the two-time Green function is performed in a standard way: $\left\langle\left\langle X^{p q} \mid X^{r s}\right\rangle\right\rangle_{q}(\omega)=K^{p q r s}\left(q, \mathrm{i} \omega_{n} \rightarrow \omega\right)$. 
In the lowest order in $t_{i j}$ in the case of $x x+y y$ polarization we should calculate (as it follows from (14))

$$
\begin{aligned}
& \left\langle T \sum_{k} \gamma_{k} \alpha_{k}^{+}(\tau) \alpha_{k}(\tau) \sum_{k^{\prime}} \gamma_{k^{\prime}} \alpha_{k^{\prime}}^{+} \alpha_{k^{\prime}}\right\rangle \\
& \gamma_{k}=\sum_{\alpha} \frac{\partial^{2} t_{k}}{\partial k_{\alpha}^{2}}=-a^{2} t_{k} \quad \text { (square lattice case) }
\end{aligned}
$$

$a$ is a lattice constant. Let us introduce unperturbed Green function

$$
\begin{aligned}
g_{i j}^{p q}(\tau) & =\delta_{i j} g^{p q}(\tau)=\delta_{i j} \frac{\left\langle T X^{p q}(\tau) X^{r s}\right\rangle}{\left\langle\left[X^{r s}, X^{p q}\right]_{ \pm}\right\rangle_{0}}, \\
g^{p q}\left(\omega_{n}\right) & =\int_{0}^{\beta} \mathrm{d} \tau \mathrm{e}^{-\mathrm{i} \omega_{n} \tau} g^{p q}(\tau)= \pm \frac{1}{\mathrm{i} \omega_{n}-\lambda_{p q}},
\end{aligned}
$$

where $\lambda_{p q}=\lambda_{p}-\lambda_{q}$; upper (lower) sign corresponds to Green function constructed on Fermi (Bose) type operators.

We use the following diagrammatic notations [21]: triangle - pq stands for Green function $g^{p q}$; circles $-p q-$ and (p) denote $\left\langle X^{p p} \pm X^{q q}\right\rangle_{0}$ and $\left\langle X^{p p}\right\rangle_{0}$ respectively; the electron transfer $\sim \sim$. Green function in Hubbard-I approximation is given by a diagrammatic series

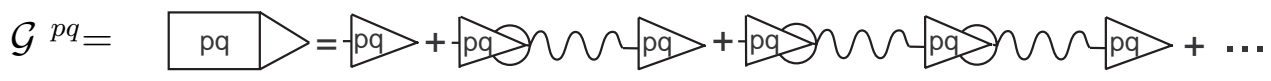

or in analytic form

$$
\mathcal{G}^{p q}=\frac{1}{\mathrm{i} \omega_{n}-\epsilon_{p q}(k)}, \quad \epsilon_{p q}(k)=\lambda_{p q}+A_{p q}\left\langle X^{p p}+X^{q q}\right\rangle t_{k},
$$

here $A_{p q}$ are coefficients, which appear due to the transformation (8), in particular $A_{\widetilde{4} \mathbf{1}}=\cos ^{2} \phi_{1}, A_{\widetilde{4} 1}=\sin ^{2} \phi_{1}$.

We consider a strong coupling case $U, g>>\sqrt{h^{2}+\Omega^{2}}>W\left(W=\sum_{j} t_{i j}\right)$ and take into account states $|1\rangle,|\widetilde{1}\rangle,|\widetilde{3}\rangle,|\widetilde{4}\rangle$ and two lowest electron subbands $\lambda_{\widetilde{4} 1}=\lambda_{\widetilde{3} 1}$, $\lambda_{\widetilde{4}}=\lambda_{\widetilde{3}}$, which are separated between themselves [9].

At the summation of diagrams we use a generalized random phase approximation (GRPA). It can be shown that the main contribution is connected with Green function $\left\langle T X^{1 \widetilde{1}}(\tau) X^{\widetilde{1} 1}\right\rangle[9]$. In diagrammatic representation we can write

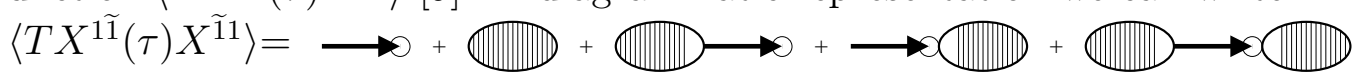

where boson Green function satisfies the Dyson equation

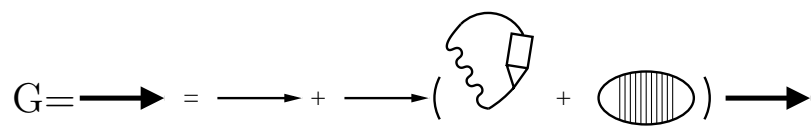

thin arrow stands for unperturbed Green function $g^{1 \tilde{1}}$. 
Similarly, for the correlator $\left\langle T \sum_{k} \gamma_{k} \alpha_{k}^{+}(\tau) \alpha_{k}(\tau) \sum_{q} \gamma_{q} \alpha_{q}^{+} \alpha_{q}\right\rangle$ we write $\frac{1}{N}\left\langle T \sum_{k} \gamma_{k} \alpha_{k}^{+}(\tau) \alpha_{k}(\tau) \sum_{q} \gamma_{k} \alpha_{q}^{+} \alpha_{q}\right\rangle=$

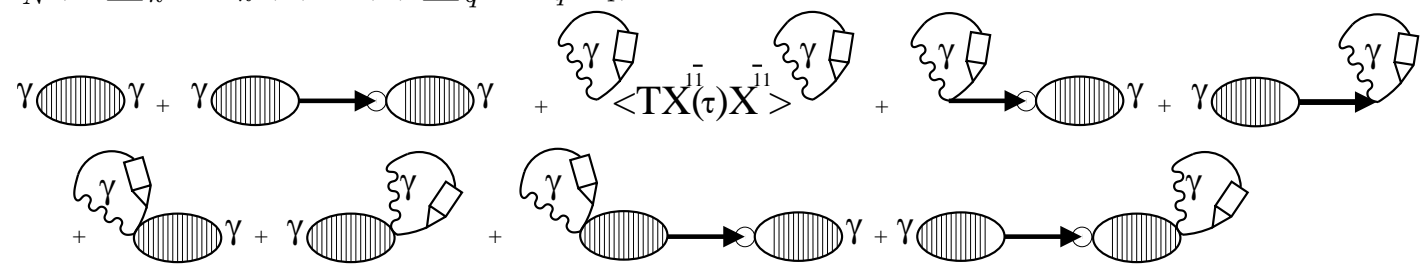

Shaded ellipses denote multi-loop diagrams, which consist of segments of the singleloop diagrams

$a=\square ; b=\bigcirc ; c=\bigcirc ; d=\square ;{ }^{\gamma} b=\gamma \bigcirc$ and similarly for loops $b^{\gamma},{ }^{\gamma} c, d^{\gamma},{ }^{\gamma} b^{\gamma}$. As it was shown in [9], the most important are diagrams

$a={ }_{\overline{\bar{p}} \boldsymbol{\overline { p }}}, p=4,3$ and similarly for $b, c, d, b^{\gamma},{ }^{\gamma} c, d^{\gamma},{ }^{\gamma} b^{\gamma}$ (with lower Green function $\mathcal{G}^{\widetilde{41}}$ or $\mathcal{G}^{\widetilde{31}}$ and upper function $\mathcal{G}^{\widetilde{41}}$ or $\left.\mathcal{G}^{\widetilde{3} 1}\right)$. In analytic form

$$
\begin{aligned}
& a=\frac{-2 \sin ^{2} \phi_{1} \cos ^{2} \phi_{1}}{N} \sum_{k} t_{k} t_{k} \frac{n\left(\epsilon_{\widetilde{4} \widetilde{1}}(k)\right)-n\left(\epsilon_{4 \widetilde{1}}(k)\right)}{i \omega_{n}+\epsilon_{\widetilde{4} \mathfrak{1}}(k)-\epsilon_{\widetilde{4} 1}(k)} \\
& b=\frac{-2}{N} \sum_{k} \frac{n\left(\epsilon_{\widetilde{41}}(k)\right)-n\left(\epsilon_{\widetilde{4} 1}(k)\right)}{\mathrm{i} \omega_{n}+\epsilon_{\widetilde{4}}(k)-\epsilon_{4 \widetilde{1}}(k)}+2 \frac{n\left(\lambda_{\widetilde{4}}\right)-n\left(\lambda_{\widetilde{4} 1}\right)}{\mathrm{i} \omega_{n}+\lambda_{\widetilde{4}}-\lambda_{\widetilde{4} 1}} \\
& c=\frac{-2 \sin ^{2} \phi_{1}}{N} \sum_{k} t_{k} \frac{n\left(\epsilon_{\widetilde{4} \tilde{1}}(k)\right)-n\left(\epsilon_{\widetilde{4} 1}(k)\right)}{\mathrm{i} \omega_{n}+\epsilon_{\widetilde{4} \widetilde{1}}(k)-\epsilon_{\widetilde{4} 1}(k)} \\
& d=\frac{-2 \cos ^{2} \phi_{1}}{N} \sum_{k} t_{k} \frac{n\left(\epsilon_{\widetilde{4} \widetilde{1}}(k)\right)-n\left(\epsilon_{\widetilde{4} 1}(k)\right)}{\mathrm{i} \omega_{n}+\epsilon_{\widetilde{4} \tilde{1}}(k)-\epsilon_{\widetilde{4} 1}(k)} .
\end{aligned}
$$

Loops ${ }^{\gamma} b, b^{\gamma},{ }^{\gamma} c, d^{\gamma},{ }^{\gamma} b^{\gamma}$ differ from $a, b, c, d$ by the presence in the sum of the multiplier $\left\langle X^{11}+X^{\widetilde{44}}\right\rangle \gamma_{k} \cos \phi_{1} \sin \phi_{1}$ for the left $\gamma$ and $\left\langle X^{\widetilde{11}}+\widetilde{X^{\widetilde{4}}}\right\rangle \gamma_{k} \cos \phi_{1} \sin \phi_{1}$ for the right $\gamma$. For example

$$
{ }^{\gamma} b=\frac{-2 \sin \phi_{1} \cos \phi_{1}}{N} \sum_{k} \gamma_{k}\left\langle X^{11}+X^{\widetilde{44}}\right\rangle \frac{n\left(\epsilon_{\widetilde{4} \widetilde{1}}(k)\right)-n\left(\epsilon_{\widetilde{4}}(k)\right)}{\mathrm{i} \omega_{n}+\epsilon_{\widetilde{4} \widetilde{1}}(k)-\epsilon_{\widetilde{4} 1}(k)}
$$

Introducing the notation

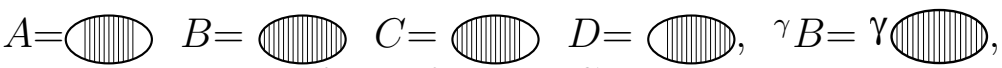

and analogously for ${ }^{\gamma} B^{\gamma}, B^{\gamma},{ }^{\gamma} C, D^{\gamma}$, we obtain the equations

$$
\left\{\begin{array}{l}
A=a+a C+d A \\
C=c+c C+b A
\end{array}\right.
$$




$$
\begin{gathered}
\left\{\begin{array}{c}
B=b+c B+b D ; \\
D=d+d D+a B,
\end{array}\right. \\
\left\{\begin{array}{l}
B^{\gamma}=b^{\gamma}+c B^{\gamma}+b D^{\gamma} ; \\
D^{\gamma}=d^{\gamma}+d D^{\gamma}+a B^{\gamma},
\end{array}\right. \\
{ }^{\gamma} B^{\gamma}={ }^{\gamma} b^{\gamma}+{ }^{\gamma} c B^{\gamma}+{ }^{\gamma} b D^{\gamma} \\
{ }^{\gamma} C={ }^{\gamma} c+{ }^{\gamma} c C+{ }^{\gamma} b A, \\
{ }^{\gamma} B={ }^{\gamma} b+{ }^{\gamma} c B+{ }^{\gamma} b D .
\end{gathered}
$$

Having solved them, we can write the expressions for the needed Green functions. For the boson Green function we obtain

$$
\begin{aligned}
G & =\frac{\left\langle X^{\widetilde{11}}-X^{11}\right\rangle}{i \omega_{n}+\lambda_{1}-\lambda_{\widetilde{1}}+\Delta-A\left\langle X^{\widetilde{1}}-X^{11}\right\rangle} \\
\Delta & =\frac{2}{N} \sum_{k} t_{k}\left[n\left(\epsilon_{\widetilde{4} 1}\right) \sin ^{2} \phi_{1}-n\left(\epsilon_{\widetilde{41}}\right) \cos ^{2} \phi_{1}\right] .
\end{aligned}
$$

The self-energy part of this function consists of two terms. The first one is connected with loop insertions into the line of unperturbed boson Green function. It leads to the renormalization of energy levels

$$
\lambda_{r} \rightarrow \widetilde{\lambda_{r}}=\lambda_{r}+\frac{1}{N} \sum_{k, p} A_{p r} t_{k} n\left(\epsilon_{p}(k)\right) .
$$

For a self-consistency, such a renormalization should be also done in the expressions for Fermi-type Green functions $\mathcal{G}^{p q}$ [17]. In our numerical calculations we do not take into account this renormalization. The second term in the self-energy part is an effective retarded interaction between pseudospins which is formed due to the band electrons.

We can write the correlator $\left\langle T X^{1 \widetilde{1}}(\tau) X^{\widetilde{1} 1}\right\rangle$ in analytic form [9]

$$
\left\langle T X^{1 \widetilde{1}}(\tau) X^{\widetilde{1} 1}\right\rangle_{q=0}=G+B+D G+G C+G D C .
$$

This expression can be rewritten in another way, which is similar to that for the transverse magnetic susceptibility of the Hubbard model [22]

$$
\begin{aligned}
\left\langle T X^{1 \widetilde{1}}(\tau) X^{\widetilde{1} 1}\right\rangle_{q=0} & =\frac{G^{0}+b}{(1-c)(1-d)-a\left(G^{0}+b\right)}, \\
\text { where } G^{0} & =\frac{\left\langle X^{\widetilde{1}}-X^{11}\right\rangle}{\mathrm{i} \omega_{n}+\lambda_{1}-\lambda_{\widetilde{1}}+\Delta} .
\end{aligned}
$$

For the correlator $\left\langle T \sum_{k} \gamma_{k} \alpha_{k}^{+}(\tau) \alpha_{k}(\tau) \sum_{q} \gamma_{q} \alpha_{q}^{+} \alpha_{q}\right\rangle$ we have

$$
\begin{aligned}
& \frac{1}{N}\left\langle T \sum_{k} \gamma_{k} \alpha_{k}^{+}(\tau) \alpha_{k}(\tau) \sum_{q} \gamma_{q} \alpha_{q}^{+} \alpha_{q}\right\rangle={ }^{\gamma} B^{\gamma}+{ }^{\gamma} C G D^{\gamma} \\
& \quad+\Delta_{\widetilde{4} 1}\left\langle T X^{1 \widetilde{1}}(\tau) X^{\widetilde{1} 1}\right\rangle_{q=0} \Delta_{\widetilde{4} \widetilde{1}}+\Delta_{\widetilde{4} 1}^{\gamma} C G+\Delta_{\widetilde{4}} D^{\gamma} G+{ }^{\gamma} B \Delta_{\widetilde{4} 1} \\
& \quad+B^{\gamma} \Delta_{\widetilde{4}}+C G D^{\gamma} \Delta_{\widetilde{4} \widetilde{1}}+{ }^{\gamma} C D G \Delta_{\widetilde{4} 1},
\end{aligned}
$$


where

$$
\Delta_{r q}=-2 \sum_{k} t_{k} n\left(\epsilon_{r q}\right) \cos \phi_{1} \sin \phi_{1}
$$

Thus, using the expression (15) we can calculate Raman scattering tensor for the case of $z z$ and $x x+y y$ polarization:

$$
\begin{aligned}
H_{k_{2}, k_{1}=0}^{z z, z z}\left(\omega_{1}, \omega_{2}\right) & =\frac{2 \omega^{\prime 2} \sin ^{2} 4 \phi_{1}\left(\frac{d_{s}}{2}\right)^{4} N}{1-\mathrm{e}^{\beta \omega}} \frac{\operatorname{Im}\left\langle\left\langle X^{1 \widetilde{1}} \mid X^{\tilde{1} 1}\right\rangle\right\rangle_{q=0}}{\omega_{2}^{2}\left(\omega_{2}-\omega^{\prime}\right)^{2}} \\
H_{k_{2}, k_{1}=0}^{x x+y y, x x+y y}\left(\omega_{1}, \omega_{2}\right) & =\frac{2(e a)^{4}}{1-\mathrm{e}^{\beta \omega}} \frac{\operatorname{Im}\left\langle\left\langle\sum_{k} \gamma_{k} \alpha_{k}^{+} \alpha_{k} \mid \sum_{q} \gamma_{q} \alpha_{q}^{+} \alpha_{q}\right\rangle\right\rangle}{\omega_{1}^{2} \omega_{2}^{2}} .
\end{aligned}
$$

At the numerical calculations we consider the case of square lattice and use the following connection between the chemical potential and electron concentration [9]

$$
\mu=\lambda_{\widetilde{4}}-\lambda_{\widetilde{1}}-W \cos ^{2} \phi_{1}\left(1-\frac{3}{2} n\right) .
$$

We assume that the dipole moment of pseudospins $d_{s}$ is smaller than the dipole momentum of electrons $d_{e}=e a$ ( $a$ is a lattice constant). This situation is typical in the case of HTSC of YBaCuO-type; in our calculations $d_{s} / d_{e}=0.6$.
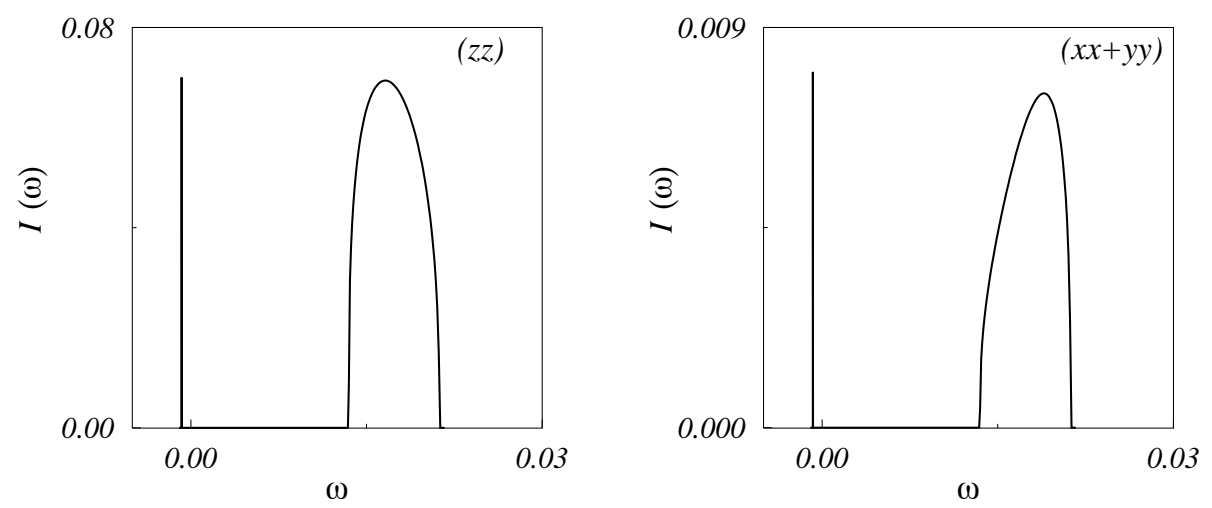

Figure 1. Raman scattering intensity for $z z$ and $x x+y y$ polarization at $T=0, W=0.1, n=0.9, h=0.16, \Omega=0.26$. Vertical lines denote deltapeak. Raman intensity is measured in arbitrary units. The frequency is shifted $\omega=\omega_{2}-\omega_{1}-\omega^{\prime}$.

From the expression (22) one can see that the imaginary part of all loops at zero temperature is different from zero only in the frequency interval

$$
-W \frac{1-\frac{3}{2} n}{1-\frac{n}{2}}<-\frac{\omega}{\cos ^{2} \phi_{1}-\frac{n}{2}}<W .
$$

The imaginary part of Green function $G$ can have a delta-peak outside this interval, because the real part of the denominator in (25) can be equal to zero [9]. 

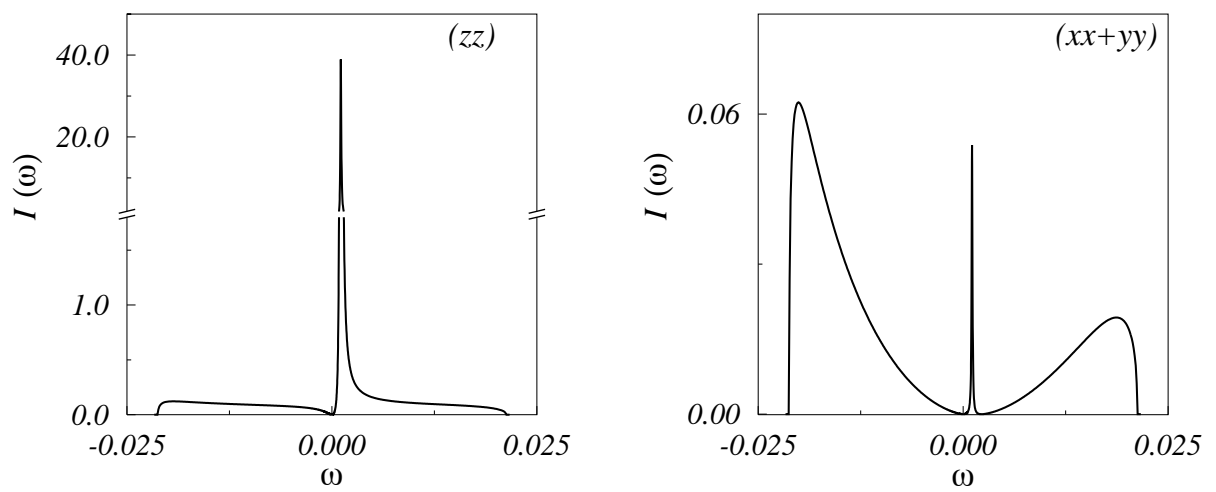

Figure 2. Raman scattering intensity for $z z$ and $x x+y y$ polarization at $T=0.015, W=0.1, n=0.9, h=0.16, \Omega=0.26$.
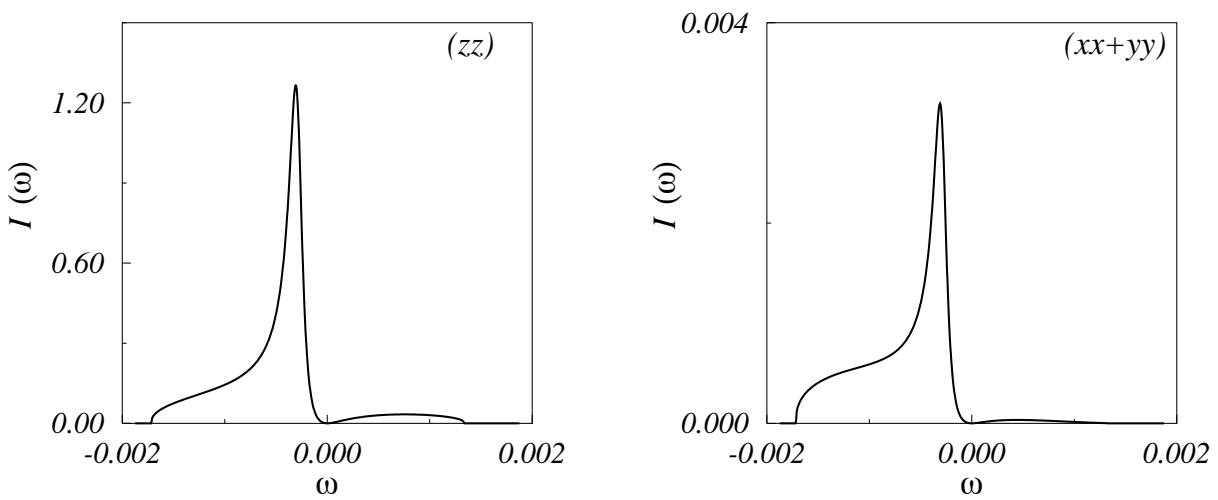

Figure 3. Raman scattering intensity for $z z$ and $x x+y y$ polarization at $T=0, W=0.1, n=0.2, h=0.25, \Omega=0.21$.

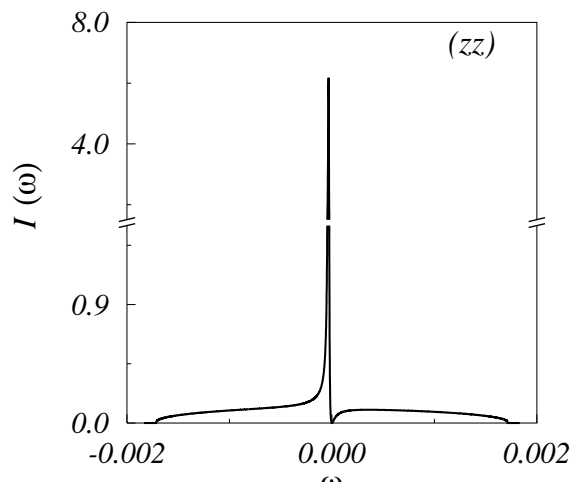

$\omega$

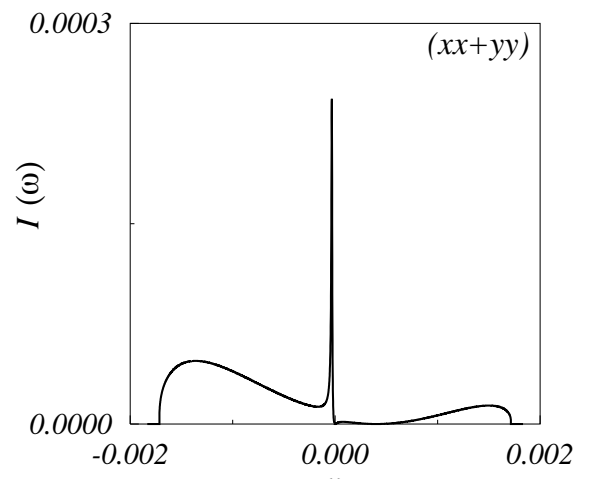

$\omega$

Figure 4. Raman scattering intensity for $z z$ and $x x+y y$ polarization at $T=0.03, W=0.1, n=0.2, h=0.25, \Omega=0.21$. 
The intensity $I(\omega) \sim H(\omega)$ for $z z$ and $x x+y y$ polarization is shown in figures 1-4 for different values of parameters of the model; frequency is shifted: $\omega=\omega_{2}-\omega_{1}-\omega^{\prime}$, $\omega^{\prime}=-\sqrt{h^{2}+\Omega^{2}}$. At $T=0$ the two components are present in the spectrum: i) the broad band with the width $\delta \omega=\left|\cos ^{2} \phi_{1}-n / 2\right| \frac{4(1-n)}{2-n} W$; ii) the narrow peak $(\delta$ function at large electron concentrations; at small concentrations this delta peak is overlapped by the band, see figures 1,3 ). The first of these components is connected with interband transitions from occupied states of $\lambda_{\widetilde{4} 1}$ subband to unoccupied ones of $\lambda_{\widetilde{4} \widetilde{1}}$ subband. The second one is coherent and is of a pseudospin origin. It is connected with collective dynamics of pseudospins (as it was mentioned, imaginary part of $G$ has a delta-peak). The effective interaction between pseudospins is formed by the electrons. Similar picture is observed for the Hubbard and $t-J$ models where the contributions from localized and itinerant magnetic moments to magnetic transverse susceptibility are observed [22].

At $T \neq 0$ for large electron concentrations, an incoherent component occupies the interval $\delta \omega=\left|\cos ^{2} \phi_{1}-n / 2\right| 2 W$ and overlaps the $\delta$-peak, see figures 2,4. function and multi-loops At the increase of temperature the Raman spectra become more symmetrical with one main peak. At high temperatures Raman spectra, calculated in GRPA are similar to those, which can be obtained when we consider the one-loop approximation (in this approximation $\operatorname{Im}\left\langle\left\langle X^{1 \widetilde{1}} \mid X^{\widetilde{1} 1}\right\rangle\right\rangle \sim \operatorname{Im} b$ and $\operatorname{Im}\left\langle\left\langle\sum_{k} \gamma_{k} a_{k}^{+} a_{k} \mid \sum_{q} \gamma_{q} a_{q}^{+} a_{q}\right\rangle\right\rangle \sim \operatorname{Im} a$, here $b$ and $a$ are one-loop contributions and their expressions are given by (22)), although central peak (at $\omega-\omega^{\prime} \sim 0$ ) in the spectrum in the case of $x x+y y$ polarization cannot be obtained in the one-loop approximation (because $\operatorname{Im} b \sim \rho\left(t^{*}\right) \delta n, \operatorname{Im} a \sim t^{* 2} \rho\left(t^{*}\right) \delta n, \rho(t)$ is a density of states, $\delta n=n\left(\epsilon_{\widetilde{4} 1}\left(t^{*}\right)\right)-n\left(\epsilon_{\widetilde{4} \tilde{1}}\left(t^{*}\right)\right), t^{*}=\frac{\omega-\omega^{\prime}}{n / 2-\cos ^{2} \phi_{1}}$ and $\left.\operatorname{Im} a\left(\omega \approx \omega^{\prime}\right) \approx 0\right)$.

From figures 1-4 one can see that the intensity of the Raman spectra for $z z$ polarization is larger than that for $x x+y y$ polarization. The obtained spectra have a complicated structure. The peak position depends on the value of $h$ parameter, which is responsible for the asymmetry of double well potential and depends, for example, on the crystal composition (that is at different doping levels in $\mathrm{YBaCuO}$ crystal case). Experimental investigations of Raman spectra in $\mathrm{YBaCuO}$ (phonon mode, connected with vibrations of apical oxygen O4) [23,24] also revealed the complex structure of spectrum, the dependence of the peak position on the doping level.

\section{Conclusions}

In this work the contributions to Raman scattering of light are investigated in the case of pseudospin-electron model. To construct the polarizability operator, the microscopic approach which is based on operator expansions is employed. Electron and pseudospin contributions to a dipole moment are taken into account. The cases of $z z$ and $x x+y y$ polarization are considered.

The presence of coherent ( $\delta$ - peak-like) and incoherent contributions, that can be distinguished at low temperatures, was established. The intensity for the case of $z z$ polarization is larger than that for the case of $x x+y y$ polarization. The obtained spectra have a complex structure; the asymmetry of lines and the dependence of their 
form on the electron concentration and temperature were established. In general, our results are in agreement with the experimental investigations of Raman spectra of $\mathrm{YBaCuO}$ (vibrations of apical oxygen) [23,24].

It should be noted, that the obtained Raman spectra can also be usefull while considering the systems with hydrogen bonds, in which the presence of double well potential is firmly confirmed. Usually such crystals appear to be dielectrics. However, complicated hydrogen-bonded complexes, which contain metallic ions and have electron conductivity have been recently revealed [25]. To our knowledge, there have been only a few experimental Raman spectrum investigations concerning such systems.

\section{Acknowledgements}

This work was supported in part by the Fundamental Research Fund of the Ministry of Ukraine for Science and Education (Project No. 02.07/266). 


\section{References}

1. Cooper S.L. Electronic and Magnetic Raman Scattering Studies of the High- $T_{\mathrm{c}}$ Cuprates. Handbook on the Physics and Chemistry of Rare Earths, vol. 31, chapter 203. Elsevier Science B.V., 2001.

2. Shastry B.S., Shraiman B.I. // Phys. Rev. Lett., 1990, vol. 65, p. 1068.

3. Freericks J.K., Devereaux T.P. //Phys. Rev. B, 2001, vol. 64, p. 125110.

4. Sarma S.D, Wang D.-W. // Phys. Rev. Lett. 1999, vol. 83, No. 4, p. 816-819.

5. Shvaika A.M, Vorobyov O, Freericks J.K, Devereaux T.P. Preprint cond-mat/0311070.

6. Stasyuk I.V., Ivankiv Ya.l., Raman scattering in crystals with ordering structure units. Preprint ITP-87-57P, Institute for Theoretical Physics, Kyiv, 1987, 25 p. (in Russian).

7. Stasyuk I.V., Mysakovych T.S., Raman scattering tensor for Hubbard and $t-J$ models. Preprint of the Institute for Condensed Matter Physics, ICMP-9-8-28E, Lviv, 1998.

8. Stasyuk I.V., Mysakovych T.S., Polarizability operator for pseudospin-electron model. Preprint of the Institute for Condensed Matter Physics, ICMP-98-34E, Lviv, 1998.

9. Stasyuk I.V., Mysakovych T.S. // Journ. Phys. Studies, 1999, vol. 3, No. 3, p. 344-358.

10. Barry R., Sharpe I.W. // Can. J. Phys., 1978, vol. 56, p. 550.

11. Falicov L.M, Kimball J.K. // Phys. Rev. Lett., 1969, vol. 22, p.997.

12. Müller K.A. // Z. Phys. B, 1990, vol. 80, p. 193.

13. Hirsch J.E., Tang S. // Phys. Rev. B., 1989, vol. 40,. p. 2179.

14. Frick M., von der Linden W., Morgenstern I., Raedt H. // Z. Phys. B - Cond. Mat., 1990, vol. 81, p. 327.

15. Stasyuk I.V., Shvaika A.M. // Condens. Matter Phys., 1994., No. 3., p. 134-175.

16. Stasyuk I.V., Havrylyuk Yu. // Condens. Matter Phys., 1999., vol. 2., p. 487.

17. Stasyuk I.V., Danyliv O.D. // Phys. Stat. Sol. B., 2000, vol. 219, p. 299.

18. Stasyuk I.V., Shvaika A.M., Tabunshchyk K.V. // Condens. Matter Phys., 1999, vol. 2, p. 109.

19. Stasyuk I.V., Mysakovych T.S. // Condens. Matter Phys., 2002, vol. 5, No. 3, p. 473491.

20. Mysakovych T.S, Stasyuk I.V. Superconductivity in pseudospin-electron model. Preprint of the Institute for Condensed Matter Physics, ICMP-03-07U, Lviv, 2003 (in Ukrainian).

21. Slobodyan P.M., Stasyuk I.V. // Teor. Mat. Fiz., 1974, vol. 19, p. 423

22. Izyumov Yu.A., Letfulov B.M. // J. Phys.: Cond. Mat., 1990, vol. 2, p. 8905.

23. Iliev M.N., Hadjiev V.G, Ivanov V.G. // Journ. Raman Spectr., 1996, vol. 27, p. 333342 .

24. Ulivi L., Zoppi M. - In: Proc. 4th Eur. Ceram. Soc. Conf., Journ. Eur. Cer. Soc., Fourth Euro Ceramics. Vol. 6, High Tc Superconductors - Part 1, p. 35-40, 1995

25. Okaniwa K., Okamoto H., Nakasuji K., Toyota J., Yamashita M. // J. Phys. Soc. Japan, 1991, vol. 60, No. 3, p. 997-1003 


\title{
Комбінаційне розсіяння світла в псевдоспін-електронній моделі для випадку сильної псевдоспін-електронної взаємодії
}

\author{
Т.С.Мисакович, І.В.Стасюк \\ Інститут фізики конденсованих систем НАН України, \\ 79011 Львів, вул. Свєнціцького, 1
}

Отримано 5 травня 2004 р.

Досліджено ангармонічні фононні внески до комбінаційного розсіяння світла у локально-ангармонічних кристалічних системах в рамках псевдоспін-електронної моделі з тунельним розщепленням рівнів. Розглянено випадок сильної псевдоспін-електронної взаємодії. Враховано псевдоспінові та електронні внески в розсіяння. Досліджено частотну залежність інтенсивності комбінаційного розсіяння світла при різних значеннях параметрів моделі та при різних співвідношеннях між поляризацією падаючого та розсіяного світла.

Ключові слова: комбінаційне розсіяння, псевдоспін-електронна модель

PACS: $71.10 \mathrm{Fd}, 71.10 . \mathrm{Fd}, 72.10 . \mathrm{Di}, 74.25 . \mathrm{Kc}$ 